\title{
Endoscopic Management of Complicated High- grade Vesicoureteral Reflux in the First Year of Life
}

\author{
(1) Ali Tekin, (1) Sibel Tiryaki, (1) İsmail Yağmur, (1) Özge Kılıç, (1) Ali Avanoğlu, (1) Ibrahim Ulman
}

Ege University Faculty of Medicine, Department of Pediatric Surgery, Clinic of Pediatric Urology, İzmir, Turkey

\begin{abstract}
Aim: The treatment of vesicoureteral reflux (VUR) in infants is controversial. Subureteric injection is considered by some to be a popular alternative to long-term antibiotic prophylaxis. In this study, we reviewed our experience in endoscopic subureteric injection to correct highgrade reflux in infants with documented indications for antireflux surgery.

Materials and Methods: The hospital records of patients with grade 4-5 VUR and breakthrough urinary tract infections who had undergone endoscopic subureteric injection in the first year of life between 2009 and 2016 were reviewed retrospectively. Radiologic success was defined as complete resolution of reflux determined via voiding cystourethrogram obtained at least three months after the injection, and clinical success was defined as the downgrading of reflux grade to below three and the absence of urinary infection.

Results: A total of 23 patients ( 5 girls, 18 boys) with 34 high-grade refluxing units were included in this study. The mean age at first injection was $6.3 \pm 1.8$ months (1-11 months). The radiologic success rate with initial injection was $61.7 \%$, and it was $85.2 \%$ after repeated injections. The overall clinical success rate after first injection was $70.6 \%$ and $97.1 \%$ after repeated injections. The mean injected material volume was $0.34 \pm 0.27(0.1-1)$ $\mathrm{mL}$ per ureter.

Conclusion: The management of high-grade infantile reflux is still controversial with insufficient data. Published studies comparing endoscopic treatment and antibiotic prophylaxis have inconclusive results due to their wide range of success rates. Although it needs to be supported by prospective studies, endoscopic treatment is a successful alternative in high-grade VUR infants with breakthrough infection.
\end{abstract}

Keywords: Vesicoureteral reflux, infant, endoscopic reflux treatment, subureteric injection, paediatric

\section{Introduction}

The management of vesicoureteral reflux (VUR) in infants, whether diagnosed with antenatal hydronephrosis or urinary infections, remains controversial. In this specific group, there are factors that complicate decision making, such as the demanding features of antireflux surgery in small babies or the possibility of spontaneous resolution even in high-grade reflux (1-3). Subureteric injection for VUR gained worldwide popularity for its easy application and short hospital stay with superior patient comfort in children, including infants (4-6). In this study, we reviewed our experience in order to evaluate the efficacy and safety of endoscopic subureteric injection to correct high-grade reflux in infants with documented indications for antireflux surgery.

\section{Materials and Methods}

The hospital records of those patients with grade 4-5 VUR and breakthrough urinary tract infections (UTIs) who had undergone endoscopic subureteric injection in the 
first year of life between 2009 and 2016 were reviewed retrospectively. We defined breakthrough UTI as a UTI with high fever $\left(>38^{\circ} \mathrm{C}\right)$ and with documented catheter collected urine culture positivity that occurred during a course of antibiotic prophylaxis. Data including patient demographics, injected material volumes, VUR grades according to their preoperative and postoperative voiding cystourethrograms (VCUG), the circumcision status of the boys, and the success rates of the treatment were recorded. Reflux was classified according to the International Reflux Study Committee's Classification System. The procedure was performed under general anesthesia using a $9.5 \mathrm{Fr} 6^{\circ}$ cystoscope (Storz, Tuttlingen Germany). Polyacrylate polyalcohol copolymer (PPC) $\left(\right.$ Vantris $^{\circledR}$, Promedon, Argentina) was administered submucosally at the 6 o'clock position of the ureteral orifice, until the creation of a prominent bulge, by use of a Williams cystoscopic injection needle (Cook Medical ${ }^{\circledR}$, Bloomington, USA). Radiologic success was defined as complete resolution of reflux determined via VCUG obtained at least three months after the injection, and clinical success was defined as the downgrading of reflux degree to below three and absence of urinary infection. The injection was repeated if persistent reflux above grade 2 was documented. Ultrasonography was performed at the postoperative first, third, and sixth months, and then annually for the followup of obstructive findings such as new onset or increased hydroureteronephrosis. This study was approved by the Ege University Medical Research Ethics Committee under the number 20-1T/47. Written informed consent was obtained from all parents of the patients.

\section{Statistical Analysis}

The chi-square test and t-test were used for statistical analyses with IBM SPSS 23.0.

\section{Results}

A total of 23 patients ( 5 girls, 18 boys) with 34 highgrade refluxing units were included in this study. The mean age at first injection was $6.3 \pm 1.8$ months (1-11 months). Four patients had contralateral low or moderate grade (1-3) reflux. There was grade 5 reflux in 27 units and grade 4 reflux in 7 units. Eleven patients had bilateral high-grade refluxing units, and 9 of them were bilateral grade 5 . The mean duration of follow-up was $69.53 \pm 24.65$ months. Continuous antibiotic prophylaxis (CAP) was started with amoxicillin for babies under three months of age, and with co-trimoxazole after this period. Ten patients who were under prophylaxis had confirmed breakthrough UTI and underwent endoscopic injection after their first breakthrough infection. In 13 patients, prophylaxis was changed to co-trimoxazole or cefixime and they were kept under observation due to the questionable compliance of their families to CAP regimens. The mean time interval from surgery to first postoperative VCUG was $5.63 \pm 0.90$ months. The radiologic success rate with initial injection was $61.7 \%$, and it was $85.2 \%$ after repeated injections. Three refluxing units at first injection and one refluxing unit at second injection were downgraded to grade 2 and grade 1 . Including these patients, the overall clinical success rate at first injection was $70.6 \%$ and $97.1 \%$ after repeated injections. The mean injected material volume was $0.34 \pm 0.27(0.1-1) \mathrm{mL}$

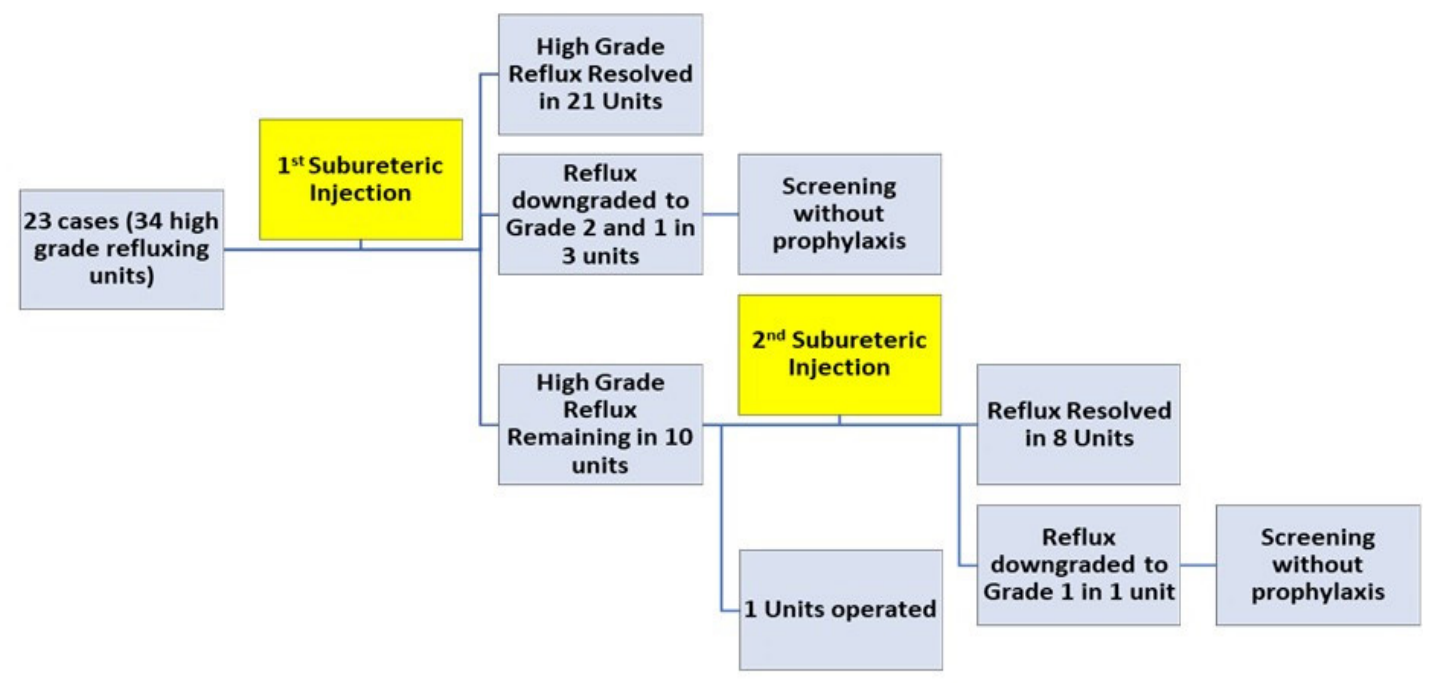

Figure 1. Flow chart of the overall management 
per ureter. There were no significant correlations between the volume of injected material and the degree of reflux, the clinical success rate or the radiological success rate (t-test, $p>0.05$ ). A flowchart depicting the steps of overall management for the study group is summarized in Figure 1.

Eleven refluxing units of 9 infants with bilateral grade 5 reflux were successfully treated at the first injection, while 6 of them required a second attempt. The overall radiologic success rate for bilateral grade 5 refluxing units was $83.3 \%$, and the clinical success rate was $100 \%$. In one patient whose reflux downgraded from 5 to 2 , antibiotic prophylaxis was terminated with no subsequent UTI.

Most of the boys (16/18) were uncircumcised, and 10 of them were circumcised during the general anesthesia given for endoscopic injection treatment. Those children of parents who did not accept circumcision and one patient with megameatus were not circumcised. When the patients were divided into two groups according to their circumcision status, there was no statistical difference in the clinical or radiological success rates between the groups (chi-square, $\mathrm{p}>0.05)$.

Eventually, only one patient required surgical correction for persistent VUR. In this case, ureteroneocystostomy was performed in line with the family's choice due to successive febrile urinary tract infections. During the open surgery, a fibrous capsule surrounding the substance was noted and successfully removed. This did not complicate ureteric dissection. No sign of obstruction, such as increased hydroureteronephrosis, was observed in any patients in a mean follow-up period of five years.

\section{Discussion}

Controversy regarding the management of VUR in the first year of life continues. The majority of VUR diagnosed in this group belong to higher grades (7). Increased risk of new scar formation in dysplastic kidneys and breakthrough infections during follow-up are strongly related to higher reflux grades (8). However, the rate of spontaneous resolution in this age group, even in highgrade reflux cases is impressive (9). Ureteroneocystostomy is a demanding surgery in infancy with an increased risk of complications due to small anatomy and fragile mucosa of the bladder. However, for a small unique group of infants with breakthrough infections as in our study group, antireflux procedures may be indicated in certain conditions like urosepsis attacks, breakthrough infections, and severe kidney damage (10). The reported series of endoscopic treatments were not promising except for a few studies
$(5,11)$. We found that the success rate in our study is higher than those of most studies that published their endoscopic injection experience in infant high-grade reflux. This promising result was the major factor that motivated us to reassess the place of endoscopic treatment in this difficult group of patients.

Several studies draw attention to a higher risk of recurrent and complicated UTI with resistant microorganisms under prophylaxis (12-14). Garin et al. (12) presented resistant bacteria in all cases of recurrent pyelonephritis with VUR under prophylactic antibiotic treatment. In a randomized controlled study, Hari et al. (13) showed similar results. In 2008, Pennesi et al. (14) reported that prophylaxis had no effect on infection and renal damage but caused recurrent UTI by resistant microorganisms in a case-control study. In a Swedish reflux trial, the trimethoprim resistant infection rate in girls under prophylaxis was higher than the endoscopic treatment and surveillance groups (15). Although the RIVUR study has shown that prophylactic antibiotics reduce the risk of UTI recurrence, the probability of resistant UTI has increased significantly (16). Following successful endoscopic treatment, we did not observe any febrile UTI except one case who eventually required ureteroneocystostomy, and resistant bacterial infection was not documented.

In 2010, a prospective study in children between 1 and 2 years of age who had grade 3 or 4 VUR was conducted by the Swedish reflux group (17). Three groups including endoscopic treatment, antibiotic prophylaxis, and followup without treatment were compared in terms of resolution of reflux, UTI, and renal scar development. The resolution rate was higher in the endoscopic treatment group than the two other groups (18). Their study showed a success rate of $71 \%$ with endoscopic treatment, excluding grade 5 cases. Another branch of their study showed no difference in UTI frequency after endoscopic treatment; however, the difference between successful and unsuccessful cases was not stated (15). The same group published another prospective study in 2016, including infants with grade 4 and 5 refluxes (19). Endoscopic treatment and prophylaxis groups were compared, and reflux resolution was $59 \%$ in endoscopic treatment, and $21 \%$ in the prophylaxis group after 1-year follow-up. The success rate after endoscopic treatment was 31\% in bilateral grade 5 reflux cases. They revealed no statistical difference between the endoscopic treatment and antibiotic prophylaxis groups regarding recurrent UTI and new renal scar formation (19). They also found that multiple recurrent infections were only seen in patients with persistent dilating reflux in follow-up. Our reflux resolution rates, including grade 5 reflux cases 
after first and repeat injections, were $63.1 \%$ and $89.4 \%$ respectively, which were higher than similar studies in the literature. In addition, we achieved an unexpectedly high success rate of $85 \%$ in bilateral grade 5 refluxes in a group with previously documented poor results with endoscopic treatment (6). None of the cases in our series had UTI in the follow-up period.

Another study which published similar results to our study in infants with moderate and severe VUR, but with a smaller group of Grade 5 reflux (3.6\%), showed a minor recurrent infection rate of $1 \%(11)$. We may speculate that the relatively low success rates of endoscopic treatment in two Swedish studies were the reason why they could not find significant difference between their prophylaxis and endoscopic treatment groups regarding urinary infection and new scar formation rates $(6,18)$. Thus, with higher success rates, endoscopic treatment could be superior to prophylaxis not only for reflux resolution but also for the prevention of recurrent UTI and new scar formation.

Long-term reflux recurrence is another issue to be considered following endoscopic treatment, which is reported to be between $13.4 \%$ and $19 \%$ within 2 years. Since our current protocol does not include routine VCUG in long term follow-up except for cases with febrile UTI, we cannot give an overall long-term recurrence rate for this study. However, a recent review of our protocol revealed a $0.9 \%$ long-term VUR recurrence in cases with recurrent febrile UTI that we obtained a VCUG for (20).

Our previous series of endoscopic VUR treatment in children of all ages showed a ureteric obstruction rate of $2.8 \%(21)$. Despite being a smaller group, this study, with similar technique and material, revealed no obstruction in a mean follow-up period of 5.7 years.

PPC is a relatively new material with particle size larger than most other materials (22-24). Our successful result is probably related to the material, which has a nonbiodegradable feature.

\section{Study Limitations}

The limitations of our study are its retrospective nature, the lack of a control group and the low number of cases. However, the high success rate in this special age group with severe reflux is noteworthy. Another issue to mention is that although the parents were informed and aware of febrile urinary tract infections, there may have been infections treated elsewhere which were not reported to us. Prospective randomized studies are required to confirm our results.
Another limitation of our study was the difficulty of excluding the possible protective effect of circumcision due to the small number of patients. Elective circumcision "might be", but so far has not proven to be a better first-line treatment in this specific, high-risk patient group. In the study by Alsaywid et al. (25), the rate of infection after the circumcision was lower than before circumcision. However, a multivariate analysis evaluating other factors that could contribute to this, such as age and the usage of prophylaxis, was missing in that study. Another study by Braga et al. (26) also states that uncircumcised status is a risk factor for febrile UTI in babies with antenatal hydronephrosis. However, they do not make any comment on circumcision as a first-line treatment for breakthrough UTI. On the other hand, in the study of Herz et al. (27), circumcision status did not affect UTI incidence in infants with antenatal hydronephrosis. Also, circumcision did not have any effect on reflux resolution.

\section{Conclusion}

The management of high-grade infantile reflux is still controversial with insufficient data. Published studies comparing endoscopic treatment and antibiotic prophylaxis have inconclusive results due to their wide range of success rates. Endoscopic treatment is a successful alternative in infants with high-grade VUR suffering breakthrough infections. Prospective randomized studies with larger numbers may support our findings.

\section{Ethics}

Ethics Committee Approval: This study was approved by the Ege University Medical Research Ethics Committee under the number 20-1T/47.

Informed Consent: Written informed consent was obtained from all parents of the patients.

Peer-review: Externally peer-reviewed.

\section{Authorship Contributions}

Design: A.T., S.T., A.A., I.U., Data Collection or Processing: A.T., I.Y., Ö.K., Analysis or Interpretation: S.T., A.A., I.U., Writing: A.T., S.T., I.Y., Ö.K., A.A., I.U.

Conflict of Interest: No conflict of interest was declared by the authors.

Financial Disclosure: The authors declared that this study received no financial support.

\section{References}

1. Scott JES. Fetal ureteric reflux: a follow-up study. Br J Urol 1993; 71:481-3. 
2. Steele BT, Robitaille P, DeMaria J, Grignon A. Follow-up evaluation of prenatally recognized vesicoureteric reflux. I Pediatr 1989; 115:95-6.

3. Gordon AC, Thomas DFM, Arthur RJ, Irving HC, Smith SEW. Prenatally diagnosed reflux: a follow-up study. Br J Urol 1990; 65:407-12.

4. Hensle TW, Grogg AL. Part 1: Vesicoureteral reflux treatment: The past, present, and future. Curr Med Res Opin 2007; 23(Suppl 4):S1-5.

5. Dawrant M), Mohanan N, Puri P. Endoscopic treatment for high grade vesicoureteral reflux in infants. I Urol 2006; 176(Suppl. 4):1847-50.

6. Nordenström J, Holmdahl G, Brandström P, et al. The Swedish infant high-grade reflux trial: Study presentation and vesicoureteral reflux outcome. J Pediatr Urol 2017; 13:130-8.

7. Tsai ID, Huang CT, Lin PY, et al. Screening high-grade vesicoureteral reflux in young infants with a febrile urinary tract infection. Pediatr Nephrol 2012; 27:955-63.

8. Olbing H, Smellie JM, Jodal U, Lax H. New renal scars in children with severe VUR: A 10-year study of randomized treatment. Pediatr Nephrol 2003; 18:1128-31.

9. Brophy MM, Austin PF, Yan Y, Coplen DE. Vesicoureteral reflux and clinical outcomes in infants with prenatally detected hydronephrosis. J Urol 2002; 168:1716-9; discussion 1719.

10. Tekgül $\mathrm{S}$, Riedmiller $\mathrm{H}$, Hoebeke $\mathrm{P}$, et al. EAU guidelines on vesicoureteral reflux in children. Eur Urol 2012; 62:534-42.

11. Puri P, Mohanan N, Menezes M, Colhoun E. Endoscopic treatment of moderate and high grade vesicoureteral reflux in infants using dextranomer/hyaluronic acid. I Urol 2007; 178(Suppl 4):1714-7.

12. Garin EH, Olavarria F, Nieto VG, Valenciano B, Campos A, Young L. Clinical significance of primary vesicoureteral reflux and urinary antibiotic prophylaxis after acute pyelonephritis: a multicenter, randomized, controlled study. Pediatrics 2006; 117:626-32.

13. Hari $P$, Hari $S$, Sinha $A$, et al. Antibiotic prophylaxis in the management of vesicoureteric reflux: a randomized doubleblind placebo-controlled trial. Pediatr Nephrol 2014; 30:479-86.

14. Pennesi M, Travan L, Peratoner L, et al; North East Italy Prophylaxis in VUR study group. Is antibiotic prophylaxis in children with vesicoureteral reflux effective in preventing pyelonephritis and renal scars? A randomized, controlled trial. Pediatrics 2008; 121:e1489-94.

15. Brandström P, Esbiörner E, Herthelius M, Swerkersson S, Jodal U, Hansson S. The Swedish reflux trial in children: iii. urinary tract infection pattern. J Urol 2010; 184:286-91.
16. Cara-Fuentes G, Gupta N, Garin EH. The RIVUR study: a review of its findings. Pediatric Nephrology 2015; 30:703-6.

17. Brandström $P$, Esbjörner $E$, Herthelius $M$, et al. The Swedish reflux trial in children: i. study design and study population characteristics. J Urol 2010; 184:274-9.

18. Holmdahl G, Brandström P, Läckgren G, et al. The Swedish reflux trial in children: ii. vesicoureteral reflux outcome. / Urol 2010; 184:280-5.

19. Nordenström I, Sjöström S, Sillén $U$, Sixt R, Brandström P. The Swedish infant high-grade reflux trial: UTI and renal damage. I Pediatr Urol 2017; 13:146-54.

20. Kilic O, Taner S, Tiryaki S, et al. Is Screening for Long-Term VUR Recurrence After Endoscopic Treatment Necessary? In: EUPSA 2019 - Belgrade, 20th Congress of European Pediatric Surgeons Association, 2019. p. 86

21. Tekin A, Yagmur I, Tiryaki S, Dokumcu Z, Ulman I, Avanoglu A. Changing bulking agent may require change in injection volume for endoscopic treatment of vesicoureteral reflux. International Braz J Urol 2018; 44:1194-9.

22. Kocherov S, Ulman I, Nikolaev $\mathrm{S}$, et al. Multicenter survey of endoscopic treatment of vesicoureteral reflux using polyacrylate-polyalcohol bulking copolymer (Vantris). Urology 2014; 84:689-93.

23. Ormaechea $M$, Ruiz E, Denes E, et al. New tissue bulking agent (polyacrylate polyalcohol) for treating vesicoureteral reflux: preliminary results in children. J Urol 2010; 183:714-7.

24. Ormaechea $M$, Paladini $M$, Pisano $R$, et al. Vantris, a biocompatible, synthetic, non-biodegradable, easy-to-inject bulking substance. Evaluation of local tissular reaction, localized migration and long-distance migration. tract infection in children with prenatal hydronephrosis: a prospective study. I Urol 2015; 193(Suppl 5):1766-71.

25. Alsaywid BS, Saleh H, Deshpande A, Howman-Giles R, Smith $\mathrm{GHH}$. High grade primary vesicoureteral reflux in boys: Longterm results of a prospective cohort study. Journal of Urology [Internet]. 2010; 184(Suppl 4):1598-603. Available from: https:// pubmed.ncbi.nlm.nih.gov/20728178/

26. Braga LH, Farrokhyar F, D'Cruz I, Pemberton J, Lorenzo AJ. Risk factors for febrile urinary tract infection in children with prenatal hydronephrosis: A prospective study. Journal of Urology [Internet]. 2015; 193:1766-71. Available from: https:// pubmed.ncbi.nlm.nih.gov/25813560/

27. Herz D, Merguerian P, McQuiston L. Continuous antibiotic prophylaxis reduces the risk of febrile UTI in children with asymptomatic antenatal hydronephrosis with either ureteral dilation, high-grade vesicoureteral reflux, or ureterovesical junction obstruction. J Pediatr Urol 2014; 10:650-4. 